\title{
On Supra-Additive and Supra-Multiplicative Maps
}

\author{
Jin Xi Chen ${ }^{1,2}$ and Zi Li Chen ${ }^{2}$ \\ ${ }^{1}$ College of Mathematics and Information Science, Shaanxi Normal University, Xi'an 710062, China \\ ${ }^{2}$ Department of Mathematics, Southwest Jiaotong University, Chengdu 610031, China
}

Correspondence should be addressed to Jin Xi Chen; jinxichen@home.swjtu.edu.cn

Received 9 May 2013; Revised 28 July 2013; Accepted 14 August 2013

Academic Editor: Gen-Qi Xu

Copyright (c) 2013 J. X. Chen and Z. L. Chen. This is an open access article distributed under the Creative Commons Attribution License, which permits unrestricted use, distribution, and reproduction in any medium, provided the original work is properly cited.

Let $A$ and $B$ be ordered algebras over $\mathbb{R}$, where $A$ has a generating positive cone and $B$ satisfies the property that $b^{2}>0$ if $0 \neq b \in B$. We give some conditions for a map $T: A \rightarrow B$ which is supra-additive and supra-multiplicative for all positive and negative elements to be linear and multiplicative; that is, $T$ is a homomorphism of algebras. Our results generalize some known results on supra-additive and supra-multiplicative maps between spaces of real functions.

Let $X$ be a compact Hausdorff space. Rădulescu [1] proved that whenever a map $T: C(X) \rightarrow C(X)$ satisfies the conditions $T(f+g) \geq T(f)+T(g)$ (supra-additive) and $T(f g) \geq$ $T(f) T(g)$ (supra-multiplicative) for all $f, g \in C(X)$, then $T$ is linear and multiplicative. Ercan [2] generalized this result to arbitrary topological spaces. For arbitrary topological spaces $X, Y$, a supra-additive and supra-multiplicative map $T$ : $C(X) \rightarrow C(Y)$ is both linear and multiplicative if and only if $\lim _{n} T\left(f^{+} \wedge n-f^{-} \wedge n\right)(y)=T(f)(y)$ for each $f \in C(X)$ and $y \in Y$ [2, Theorem 2]. Similar results were obtained for rings (see $[3,4])$. In [3] Dhombres showed that if $A$ is a ring and $R$ is an ordered ring in which nonzero elements have nonzero positive squares, the supra-additive and supra-multiplicative map $T: A \rightarrow R$ is both additive and multiplicative, that is, a homomorphism of rings. Recently, Gusić [5] considered supra-additive and supra-multiplicative maps between ordered fields. It was proved that on ordered fields every supra-additive and supra-multiplicative nonzero map is an injective homomorphism of fields.

Let $A$ and $B$ be ordered algebras over $\mathbb{R}$, where $A$ has a generating positive cone and $B$ satisfies the property that $b^{2}>$ 0 if $0 \neq b \in B$. In this short paper, we prove that if a positive map $T: A \rightarrow B$ is supra-additive and supra-multiplicative for all positive and negative elements in $A$, then $T$ is indeed linear and multiplicative. In particular, if $A$ is squareroot closed, then every map which is supra-additive and supramultiplicative for all positive and negative elements is both linear and multiplicative. From our result, it follows that for arbitrary topological spaces $X, Y$, a supra-additive and supramultiplicative map $T: C(X) \rightarrow C(Y)$ is indeed both linear and multiplicative. This generalizes the results of Rădulescu [1] and Ercan [2]. As a special case we consider the supraadditive and supra-multiplicative maps on $C_{0}(X)$ and obtain a Banach-Stone type result.

Recall that an ordered real vector space $A$ under a multiplication is said to be an ordered algebra whenever the multiplication makes $A$ an algebra, and in addition it satisfies the following property: if $a_{1}, a_{2} \geq 0$, then $a_{1} a_{2} \geq 0$. A is called Archimedean if $x, y \in A$ and $n x \leq y$ for all $n \in \mathbb{N}$ implies that $x \leq 0$. The set $A^{+}=\{a \in A: a \geq 0\}$ is called the positive cone of $A$. The positive cone of $A$ is said to be generating (or $A$ is positively generated) if $A=A^{+}-A^{+}$. A map $T: A \rightarrow B$ between two ordered algebras is called positive whenever $T\left(A^{+}\right) \subseteq B^{+}$. Let $A^{-}:=-A^{+}$and $A^{ \pm}:=A^{+} \cup A^{-}$.

Theorem 1. Let $A$ be an ordered algebra which has a generating positive cone. Let $B$ be an Archimedean ordered algebra satisfying the property that $b^{2}>0$ if $0 \neq b \in B$. If a positive map $T$ : $A \rightarrow B$ satisfies the following inequalities:

(1) $T\left(a_{1}+a_{2}\right) \geq T\left(a_{1}\right)+T\left(a_{2}\right)$ (supra-additive),

(2) $T\left(a_{1} a_{2}\right) \geq T\left(a_{1}\right) T\left(a_{2}\right)$ (supra-multiplicative),

for all $a_{1}, a_{2} \in A^{ \pm}$, then $T$ is both linear and multiplicative. 
Proof. From the inequality $T(0)=T(0+0) \geq T(0)+T(0)$, it follows that $T(0) \leq 0$. On the other hand, $T(0) \geq T(0 \cdot 0) \geq$ $T(0) T(0) \geq 0$. Therefore, $T(0)=0$. It should be noted that $a^{2} \geq 0$ for every $a \in A^{ \pm}$. By the supra-additivity of $T$, we have

$$
0=T(0)=T\left(a^{2}-a^{2}\right) \geq T\left(a^{2}\right)+T\left(-a^{2}\right)
$$

for every $a \in A^{ \pm}$. Thus, for every $a \in A^{ \pm}$, from the following inequalities:

$$
\begin{aligned}
0 \leq & {[T(a)+T(-a)]^{2}=T(a)^{2}+T(a) T(-a) } \\
& +T(-a) T(a)+T(-a)^{2} \\
\leq & T\left(a^{2}\right)+T\left(-a^{2}\right)+T\left(-a^{2}\right)+T\left(a^{2}\right) \\
= & 2\left[T\left(a^{2}\right)+T\left(-a^{2}\right)\right] \leq 0,
\end{aligned}
$$

it follows that $[T(a)+T(-a)]^{2}=0$ for every $a \in A^{ \pm}$. By our hypothesis on $B$, we have $T(-a)=-T(a)$ for all $a \in A^{ \pm}$. Now, for all $a_{1}, a_{2} \in A^{ \pm}$, we have

$$
\begin{aligned}
T\left(a_{1}+a_{2}\right) & \geq T\left(a_{1}\right)+T\left(a_{2}\right) \\
& =-T\left(-a_{1}\right)-T\left(-a_{2}\right) \\
& =-\left[T\left(-a_{1}\right)+T\left(-a_{2}\right)\right] \\
& \geq-T\left(-a_{1}-a_{2}\right) \\
& =T\left(a_{1}+a_{2}\right) .
\end{aligned}
$$

That is, $T\left(a_{1}+a_{2}\right)=T\left(a_{1}\right)+T\left(a_{2}\right)$ for all $a_{1}, a_{2} \in A^{ \pm}$. Similarly, for all $a_{1}, a_{2} \in A^{ \pm}$, from

$$
\begin{aligned}
T\left(a_{1} a_{2}\right) & \geq T\left(a_{1}\right) T\left(a_{2}\right)=-T\left(a_{1}\right) T\left(-a_{2}\right) \\
& \geq-T\left(-a_{1} a_{2}\right)=T\left(a_{1} a_{2}\right)
\end{aligned}
$$

it follows that $T\left(a_{1} a_{2}\right)=T\left(a_{1}\right) T\left(a_{2}\right)$ for all $a_{1}, a_{2} \in A^{ \pm}$.

Next, because $A$ is positively generated and $B$ is Archimedean, by the positive additivity of $T$ on $A^{+}$and the Kantorovich theorem (cf. [6, Proposition 1, page 150] or [7, Theorem 1.7]) there exists a unique positive linear map $S$ : $A \rightarrow B$ such that $S=T$ on $A^{+}$, where $S$ is defined by $S\left(a_{1}-\right.$ $\left.a_{2}\right)=T\left(a_{1}\right)-T\left(a_{2}\right)$ for all $a_{1}, a_{2} \in A^{+}$. For every $a \in A$, there exist $u, v \in A^{+}$satisfying $a=u-v$ since $A$ is positively generated. From

$$
\begin{aligned}
T(a) & =T(u-v)=T(u)+T(-v) \\
& =T(u)-T(v) \\
& =S(u-v)=S(a),
\end{aligned}
$$

it follows that $T=S$ on $A$. This implies that $T$ is linear. On the other hand, let $a_{1}$ and $a_{2}$ be arbitrary elements in $A$. Since $A^{+}$ is positively generated, there exist $u_{i}, v_{i} \in A^{+}(i=1,2)$, such that $a_{1}=u_{1}-v_{1}$ and $a_{2}=u_{2}-v_{2}$. We have

$$
\begin{aligned}
T\left(a_{1} a_{2}\right)= & T\left[\left(u_{1}-v_{1}\right)\left(u_{2}-v_{2}\right)\right] \\
= & T\left(u_{1} u_{2}\right)-T\left(u_{1} v_{2}\right)-T\left(v_{1} u_{2}\right)+T\left(v_{1} v_{2}\right) \\
= & T\left(u_{1}\right) T\left(u_{2}\right)-T\left(u_{1}\right) T\left(v_{2}\right) \\
& -T\left(v_{1}\right) T\left(u_{2}\right)+T\left(v_{1}\right) T\left(v_{2}\right) \\
= & {\left[T\left(u_{1}\right)-T\left(v_{1}\right)\right]\left[T\left(u_{2}\right)-T\left(v_{2}\right)\right] } \\
= & T\left(a_{1}\right) T\left(a_{2}\right) .
\end{aligned}
$$

That is, $T$ is multiplicative, as desired.

Remark 2. Let $A$ and $B$ be as in the above theorem. It may be asked whether the positive map $T: A \rightarrow B$ is linear and multiplicative whenever $T: A \rightarrow B$ is supra-additive and supramultiplicative only on $A^{+}$or only on $A^{-}$. Indeed, this is not the case. For instance, the positive nonlinear map $f: \mathbb{R} \rightarrow \mathbb{R}$ defined by $f(x)=|x|$ is additive and multiplicative on $\mathbb{R}^{+}$(or $\mathbb{R}^{-}$, resp.).

Recall that an ordered algebra $A$ is said to be squareroot closed and that whenever for any $a \in A^{+}$there exists $x \in A^{+}$, such that $a=x^{2}$. When $A$ is square-root closed, we have the following corollary.

Corollary 3. Let $A$ be a square-root closed ordered algebra with a generating positive cone. Let $B$ be an Archimedean ordered algebra satisfying the property that $b^{2}>0$ if $0 \neq b \in$ $B$. If a map $T: A \rightarrow B$ is supra-additive and supra-multiplicative on $A^{ \pm}$, then $T$ is both linear and multiplicative on $A$.

Proof. By the above Theorem, to complete the proof, we need only to verify that $T$ is positive. Since $A$ is square-root closed for each $a \geq 0$ in $A$ there exists $x \in A^{+}$, such that $a=x^{2}$. Hence, by our hypothesis on $B$, we have

$$
T(a)=T\left(x^{2}\right) \geq T(x)^{2} \geq 0 .
$$

This implies that $T$ is positive.

Remark 4. It should be noted that the space of all real functions (all real continuous functions) on a nonempty set (a topological space, resp.), with the pointwise algebraic operations and the pointwise ordering, is a square-root closed Archimedean lattice-ordered algebra with the property mentioned in Corollary 3. Thus, the results on supra-additive and supra-multiplicative maps between spaces of real functions obtained by Rǎdulescu [1], Volkmann [4], and Ercan [2] can now follow from Corollary 3. In their earlier proofs, the constant function or the multiplicative unit element plays an essential role.

Let $X$ be a locally compact Hausdorff space, and let $C_{0}(X)$ be the Banach lattice of all continuous real functions defined on $X$ and vanishing at infinity. Note that $C_{0}(X)$ does not necessarily contain the constant function or a unit element 
unless $X$ is compact. The following result is an immediate consequence of Corollary 3.

Corollary 5. Let $X$ and $Y$ be locally compact Hausdorff spaces. If $T: C_{0}(X) \rightarrow C_{0}(Y)$ is supra-additive and supramultiplicative for all elements in $C_{0}(X)^{ \pm}$, then $T$ is an algebra and lattice homomorphism.

Recall that $T: C_{0}(X) \rightarrow C_{0}(Y)$ for any $f, g$ is said to be separating or disjointness preserving if, for any $f, g \in C_{0}(X)$, $f g=0$ implies that $T f T g=0$. Clearly, every multiplicative map is disjointness preserving. Combining Corollary 5 with Theorem 2 of [8] or Theorem 8 in [9], we obtain the following Banach-Stone type result.

Corollary 6. Let $X, Y$ be locally compact Hausdorff spaces. If there exists a bijection from $C_{0}(X)$ onto $C_{0}(Y)$ which is supra-additive and supra-multiplicative on $C_{0}(X)^{ \pm}$, then $X$ is topologically homeomorphic to $Y$.

\section{Acknowledgment}

The authors would like to thank the reviewer for his/her kind comments and valuable suggestions which have improved this paper. The authors were supported in part by the Fundamental Research Funds for the Central Universities (SWJTU11CX154, SWJTU12ZT13) and NSFC (No. 11301285).

\section{References}

[1] M. Rădulescu, "On a supra-additive and supra-multiplicative operator of $C(X)$," Bulletin Mathématique de la Société des Sciences Mathématiques de la République Socialiste de Roumanie, vol. 24, no. 3, pp. 303-305, 1980.

[2] Z. Ercan, "A remark on supra-additive and supra-multiplicative operators on $C(X)$," Mathematica Bohemica, vol. 132, no. 1, pp. 55-58, 2007.

[3] J. Dhombres, "Sur les fonctions simultanément suradditives et surmultiplicatives," Comptes Rendus Mathématiques. La Société Royale du Canada. L'Academie des Sciences, vol. 5, no. 5, pp. 207210, 1983.

[4] P. Volkmann, "Sur les fonctions simultanément suradditives et surmultiplicatives," Bulletin Mathématique de la Société des Sciences Mathématiques de la République Socialiste de Roumanie, vol. 28, no. 2, pp. 181-184, 1984.

[5] I. Gusić, "A note on certain maps between ordered fields," Journal of Mathematical Inequalities, vol. 3, no. 4, pp. 657-661, 2009.

[6] R. Cristescu, Topological Vector Spaces, Editura Academiei, Bucharest, Romania, 1977.

[7] C. D. Aliprantis and O. Burkinshaw, Positive Operators, vol. 119 of Pure and Applied Mathematics, Academic Press, Orlando, Fla, USA, 1985.

[8] J. J. Font and S. Hernández, "On separating maps between locally compact spaces," Archiv der Mathematik, vol. 63, no. 2, pp. 158-165, 1994.

[9] J.-S. Jeang and N.-C. Wong, "Weighted composition operators of $C_{0}(X)$ 's," Journal of Mathematical Analysis and Applications, vol. 201, no. 3, pp. 981-993, 1996. 


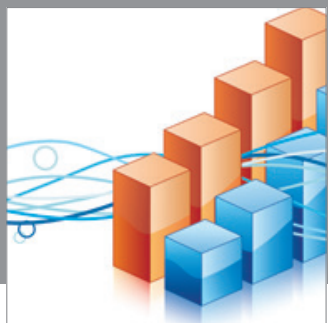

Advances in

Operations Research

mansans

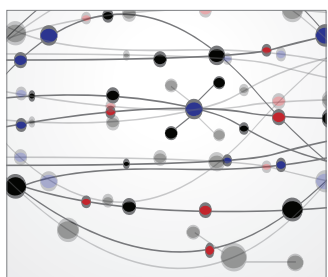

The Scientific World Journal
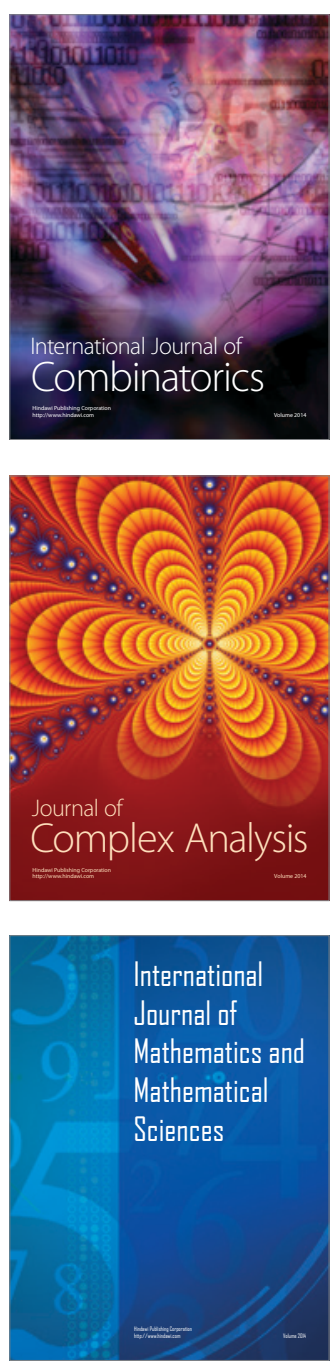
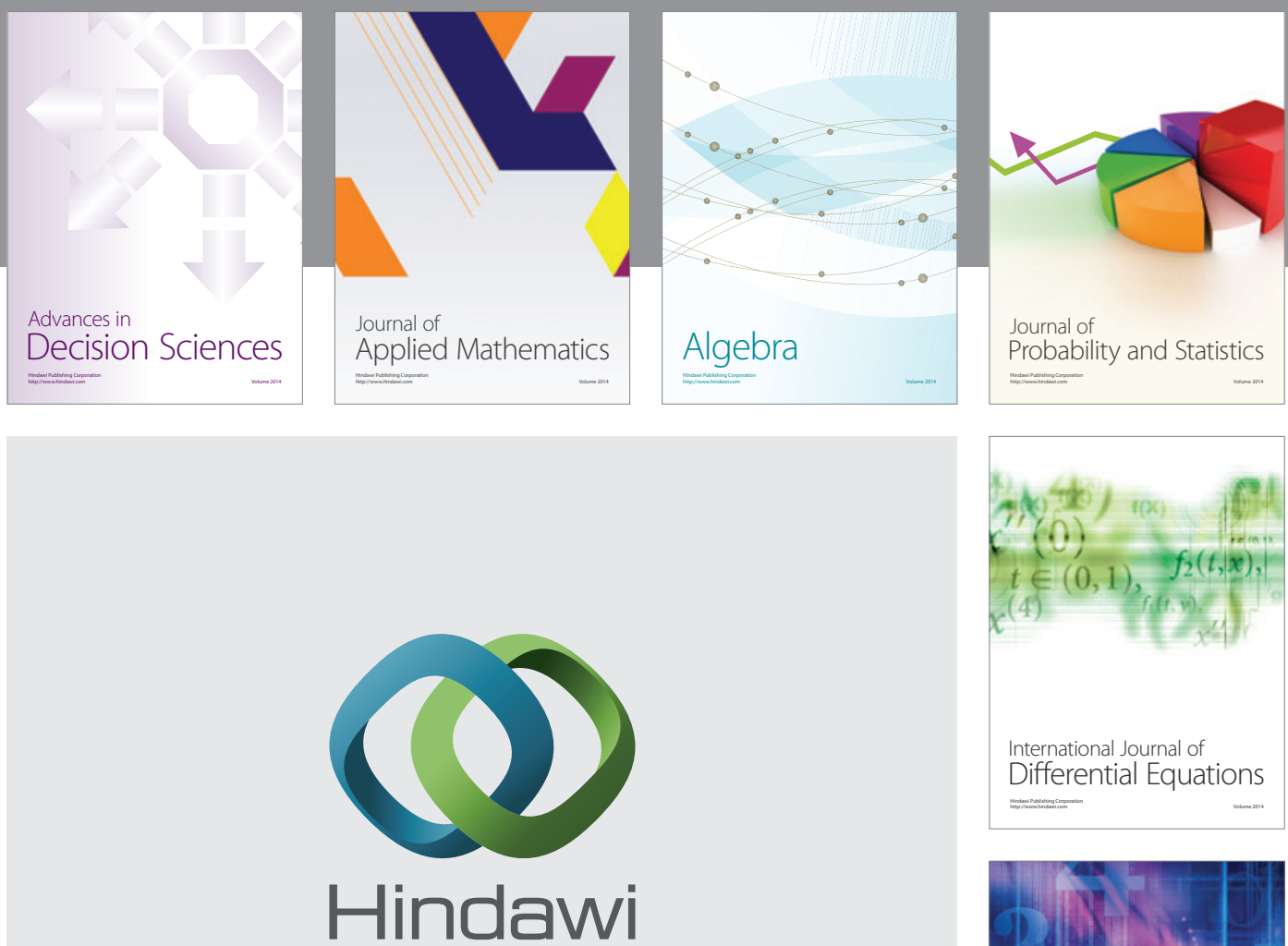

Submit your manuscripts at http://www.hindawi.com
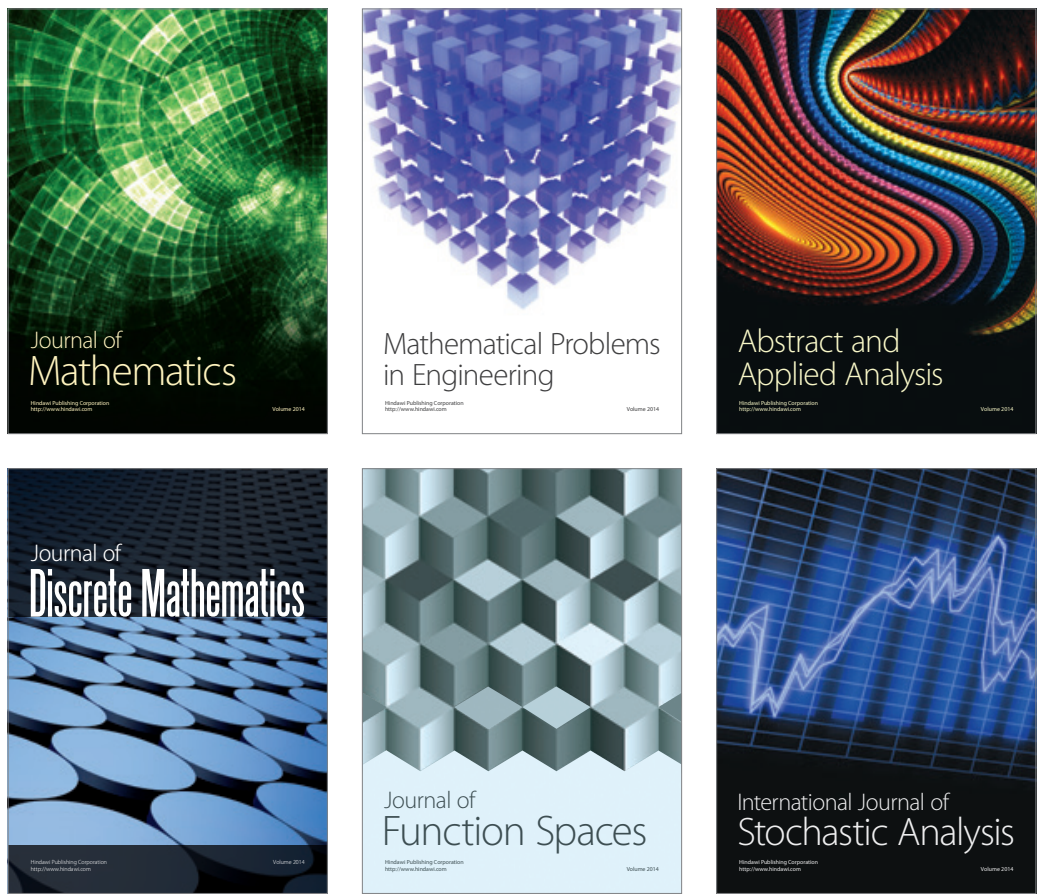

Journal of

Function Spaces

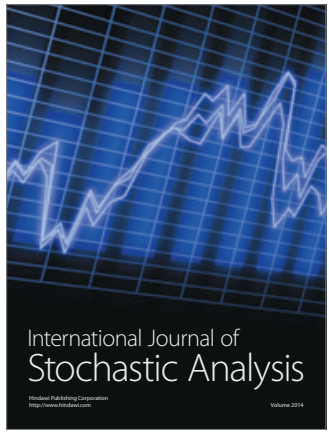

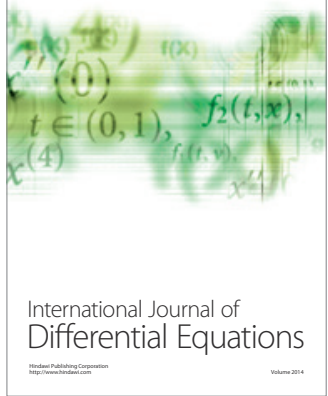
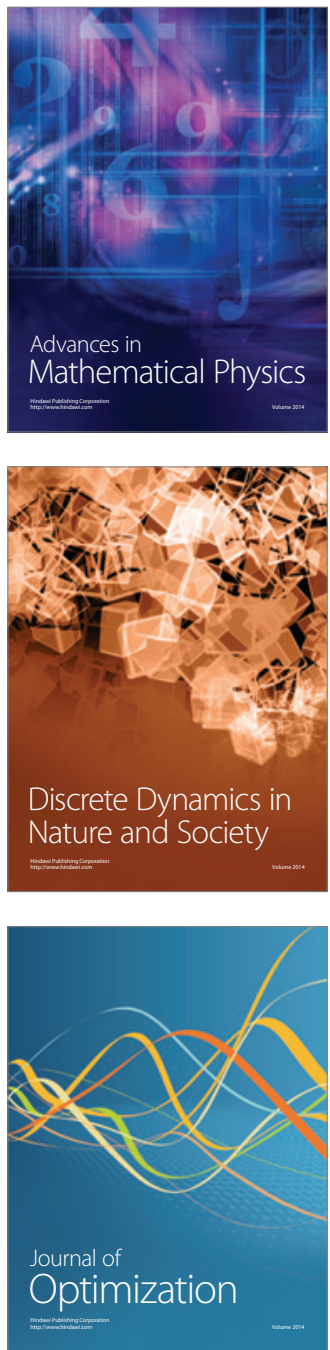